\title{
The Value of Rate Regularity and Multiplicity Measures to Detect Ventricular Tachycardia in the Presence of Atrial Fibrillation or Flutter
}

\author{
CHIH-MING JAMES CHIANG, JANICE M. JENKINS, \\ and LORENZO A. DICARLO \\ From the Department of Electrical Engineering and Computer Science School of Engineering, The \\ University of Michigan; and The Michigan Heart and Vascular Institute and Cardiac \\ Electrophysiology Laboratory, St. Joseph Mercy Hospital, Ann Arbor, Michigan
}

CHIANG, C-M.J., ET AL.: The Value of Rate Regularity and Multiplicity Measures to Detect Ventricular Tachycardia in the Presence of Atrial Fibrillation or Flutter. The predominant cause of inappropriate therapy by implantable antitachycardia devices with pacing and nonpacing cardioverter defibrillators, is mistaking a fast ventricular response during atrial fibrillation or flutter with true ventricular tachycardia (VT). The distinction between these arrhythmias is an important consideration in addressing the problem of reducing false-positives in detection mechanisms for implantable devices. Dual chamber analysis that examines atrial and ventricular event ratios has been proposed as a solution to this problem, but would still fail in distinguishing paroxysmal VT requiring treatment from a fast but otherwise benign ventricular response during atrial fibrillation or flutter. In this study, two methods for discriminating these tachyarrhythmias were evaluated. Method 1 examined ventricular rate and rate regularity as a method for VT detection. Method 2 combined rate and regularity as well as an additional multiplicity criterion for recognition of atrial flutter with a fast ventricular response. In 20 patients, Method 1 had $100 \%$ sensitivity of VT detection and $80 \%$ specificity for detection of atrial fibrillation or flutter. Method 2 had $90 \%$ sensitivity and $90 \%$ specificity. These results suggest that use of these algorithms in future implantable devices would result in a decrease in false-positive device therapies. (PACE 1994; 17:1503-1508)

ventricular tachycardia, arrhythmia detection, atrial fibrillation, antitachycardia devices

\section{Introduction}

Antitachycardia devices (ATDs) have become increasingly popular and effective methods for prevention of sudden cardiac death due to ventricular tachycardia (VT) and ventricular fibrillation (VF). Among ATDs are implantable cardioverter defibrillators (ICDs) and the newer generation

This work was partially supported by a grant from Medtronic Corporation, Intel Corp., and National Science Foundation Grants BCS-8909042 and EID-9023514.

Address for reprints: Chih-ming J. Chiang, Ph.D., Telectronics Pacing Systems, 7400 S. Tucson Way, Englewood, CO 80112. Fax: (303) 799-2213.

Received July 15, 1993; revision January 10, 1994; accepted February 3, 1994. models capable of programmed stimulation in addition to cardioversion and defibrillation. Over 43,000 ICDs ${ }^{1,2}$ have been implanted with impressive survival results. ${ }^{1,3}$ However, these devices use one-channel ventricular rate-based detection schemes $^{4,5}$ and false-positive detection of VT and VF in the presence of atrial tachyarrhythmias remains a major source of inappropriate therapy. ${ }^{6-11}$ In particular, the major cause of false-positive delivery of shock is mistaking atrial fibrillation/flutter with a fast ventricular response as VT. ${ }^{8-11}$ Delivery of inappropriate therapy is not of trivial consideration. False-positive therapy has been observed to induce VT and VF with potentially serious consequences. ${ }^{12,13}$

Two-chamber analysis was proposed over a decade ago as a means of improving arrhythmia 
analysis. ${ }^{14,15}$ The hallmark of two-chamber analysis includes a predominance of atrial events indicating supraventricular tachycardia (SVT), and ventricular events in excess of atrial reflecting ventricular arrhythmias. However, such discriminators fail in the case of distinguishing true VT from fast ventricular response due to competing atrial fibrillation or flutter. With classic dual chamber logic, where the faster chamber takes precedence, competing VTs would typically be classified as atrial fibrillation or flutter, with potentially serious consequences for the patient. Alternative criteria need to be included to resolve this problem where competing atrial and ventricular rhythms are present. In this study, two new methods that utilize rate metrics from atrial and ventricular channels were evaluated for separating fast ventricular response in atrial fibrillation/flutter from true VT.

\section{Methods}

Two variations of two-chamber algorithms were developed that examine atrial and ventricular rate, rate regularity, and rate multiplicity, and combine this information heuristically into diagnostic schemes that attempt to discern VT in the presence of concurrent atrial fibrillation or flutter.

Logic for the algorithms was based on electrophysiological considerations. First, ventricular conduction pattern in atrial fibrillation/flutter is typically irregular, with a large variance in cycleto-cycle ventricular intervals. Observation of such irregularity of ventricular conduction was combined with underlying ventricular rate to exclude the existence of VT during atrial fibrillation or flutter.

The first of the two methods utilized the above information and examined atrial rate, ventricular rate, and ventricular rate regularity. Initially, a fast atrial rate was classified into either atrial fibrillation ( $>330 \mathrm{cycles} / \mathrm{min}$ ) or atrial flutter ( $>240 \mathrm{cy}$ cles/min). The ventricular rate was assessed concurrently. If the ventricular rate in the presence of a fast atrial rate exceeded the tachycardia threshold of 110 cycles/min, the possibility of concurrent VT existed and the algorithm examined for regularity in the ventricular chamber. Regularity was defined as a threshold difference $x$ falling below the minimum cycle length and the median cycle length of nine consecutive ventricular cycles, i.e.,

$$
\text { Regularity }=\frac{\text { Median }- \text { Minimum }}{\text { Median }} \leq x \%
$$

Irregularity for this algorithm was defined as a difference greater than the threshold defined above. A nine interval median was chosen based upon empirical considerations. If ventricular rate was both regular and above the VT rate threshold, the diagnosis was VT, otherwise atrial fibrillation or flutter was diagnosed by default.

An additional electrophysiological consideration utilized in the second algorithm and not the first method addressed atrial flutter, which is often characterized by regular atrial depolarization. In this situation, conduction to the ventricles might also exhibit regularity as in the case of $2: 1,3: 1$, or $\mathrm{n}: \mathrm{l}$ conduction patterns. Regularity of ventricular response could, therefore, be a confounding factor. Therefore, an additional criterion, termed the multiplicity measure, was utilized when atrial flutter was detected to further refine the discrimination of VT occurrence.

Thus, A:V rate multiplicity, as well as atrial rate, ventricular rate, and ventricular rate regularity, were combined for recognition and distinction of competing rhythms in Method 2. A flow chart of Method 2 is shown in Figure 1. In the presence of atrial fibrillation, the diagnosis was VT if the ventricular rate exceeded the VT rate threshold and exhibited regularity. In the presence of atrial flutter, if ventricular regularity and VT rate were satisfied, a multiplicity rule was applied. Multiplicity is defined as Median Ventricular Interval (MVI) MODULUS Median Atrial Interval (MAI) being less than $x \%$ or greater than $(100-x) \%$ of the median atrial interval. Between $x$ and $(100-$ $x) \%$, the atrial rate was not considered to be a multiple of the ventricular rate. The formula is given as:

$$
x \% \leq \frac{(M V I) M O D(M A I)}{M A I} \leq(100-x) \%
$$

If the multiplicity measure was not satisfied, the diagnosis was VT. Otherwise, a regular atrial flut- 


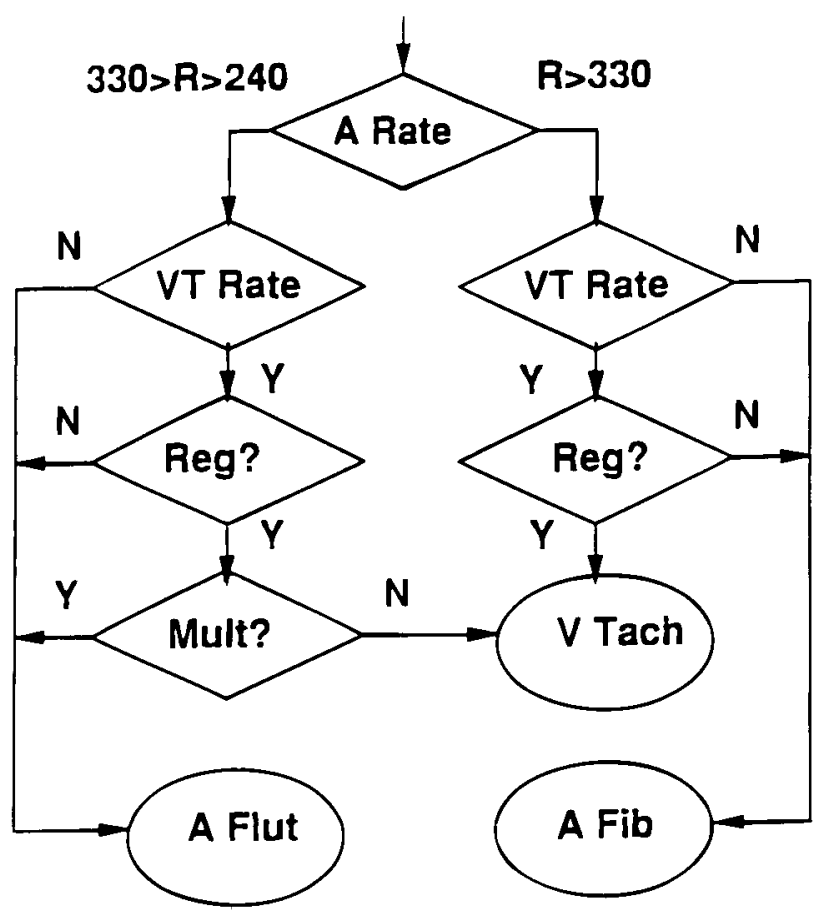

Figure 1. Diagnosis diagram of ventricular tachycardia in atrial fibrillation ( $A$ Fib) or atrial flutter ( $A$ Flut). $R$ = rate; $A=$ atrial; $V=$ ventricular; Tach = tachycardia $\operatorname{Reg}=$ regularity; Mult $=$ multiplicity.

ter with regular ventricular response was diagnosed.

For determining success or failure in classifying each passage of arrhythmia, a broader contextual diagnosis was utilized in which the predominant diagnosis of the last eight ventricular cycle diagnoses (6/8) was chosen as the true arrhythmia diagnosis. This was done to prevent isolated erroneous diagnoses from affecting a correct continuing rhythm identification. Therefore, occasional isolated VT diagnoses between a continuing stream of atrial fibrillation diagnoses would be classified as atrial fibrillation instead of VT.

\section{Materials}

The two algorithms were tested using 20 rhythm passages from 17 patients: 10 atrial fibrillation and/or flutter with fast ventricular response, and 10 atrial fibrillation and/or flutter with competing VT. Patient electrograms were recorded from endocardial bipolar electrodes $(1 \mathrm{~cm})$ in the high right atrium and right ventricular apex onto FM magnetic tape (model 3968A, Hewlett-Packard, San Diego, CA, USA) at 3/3/4 inches/sec (Ann Arbor Electrogram Libraries, Ann Arbor, MI, USA). Data were submitted to real-time computer diagnosis from custom software developed inhouse. Analysis was performed on an 80486 system with clock speed of $50 \mathrm{MHz}$ (Intel X486/50E). Data were digitized at $1,000 \mathrm{~Hz}$ per channel at 12 bit analog-to-digital resolution. Sensing was accomplished via a trigger that is adaptable to varying waveform amplitudes. ${ }^{16}$

Statistics of the patient cases are given in Table I. In 14 of 20 patients atrial flutter occurred, and in 6 of 20 atrial fibrillation. Ten had concurrent VT and ten cases exhibited fast ventricular response as determined by an expert cardiologist.

Table I.

Diagnosis of VT with Competing AF or AFL Versus AF or AFL with Fast Ventricular Response

\begin{tabular}{lcc}
\hline Patient & $\begin{array}{c}\text { True A Rhythm } \\
\text { (AFL or AF) }\end{array}$ & $\begin{array}{c}\text { True V Rhythm } \\
\text { (VT or Fast VR) }\end{array}$ \\
\hline AAEL 133 & AFL & Fast VR \\
AAEL 133 & AF & VT \\
AAEL 139 & AF/AFL & VT \\
AAEL 139 & AF & VT \\
AAEL 173 & AFL & VT \\
AAEL 182 & AFL & Fast VR \\
AAEL 182 & AFL & VT \\
AAEL 208 & AF & VT \\
AAEL 224 & AFL & VT \\
AAEL 239 & AF & Fast VR \\
AAEL 239 & AF & VT \\
AAEL 244 & AFL & Fast VR \\
AAEL 245 & AF/AFL & Fast VR \\
AAEL 288 & AF/AFL & Fast VR \\
AAEL 288 & AFL & VT \\
AAEL 337 & AFL & Fast VR \\
AAEL 340 & AFL & Fast VR \\
AAEL 342 & AF/AFL & Fast VR \\
AAEL 352 & AF & Fast VR \\
AAEL 366 & AFL & VT \\
\hline & 6 AF & 10 Fast VR \\
Total $=20$ & AF/AFL & 10 VT \\
\hline
\end{tabular}

$A=$ atrial; $A F=$ atrial fibrillation; $A F L=$ atrial flutter; Fast $V R=$ fast ventricular response; $\mathrm{Pt}=$ Ann Arbor Electrogram Libraries patient number; $V=$ ventricular; $V T=$ ventricular tachycardia. 


\section{Results}

Overall results from Methods 1 and 2, the first without and the second with the multiplicity rule, are presented in Tables II and III, respectively. The Tables contain results from varying the regularity and multiplicity thresholds. For Method 1, which utilized ventricular rate and ventricular regularity measures only, there was a tradeoff between detection of VT (sensitivity) and recognition of atrial fibrillation and flutter (specificity) when the regularity threshold value changed. A $10 \%$ threshold gave the highest number correct out of the total of 20 passages (18/20 cases correct), with $100 \%$ VT identification and $80 \%$ atrial fibrillation/flutter detection. In Method 2, which incorporated a multiplicity criterion as well as rate and regularity, a $10 \%$ threshold for the multiplicity and regularity criteria gave best performance with 18 of 20 (90\% VT diagnosis, $90 \%$ atrial fibrillation flutter detection) correct. Method 2 achieved slightly better atrial fibrillation/flutter detection at the expense of a slight decrease in VT detection.

\section{Discussion}

For Method 1 (with a $10 \%$ regularity criterion), one of the two errors was an atrial fibrillation/flutter passage with runs of ventricular premature depolarizations (VPDs) with regular cycles, while the second was an atrial flutter with $2: 1$ conduction with ventricular rate regularity. For Method 2 (with $10 \%$ regularity and $10 \%$ multiplicity criterion), there was one VT misdiagnosed as atrial flutter and one atrial flutter falsely diagnosed

\section{Table II.}

Results of Method 1

\begin{tabular}{ccc}
\hline Regularity Threshold & Sensitivity & Specificity \\
\hline $2.5 \%$ & $10 \%$ & $100 \%$ \\
$5.0 \%$ & $60 \%$ & $90 \%$ \\
$7.5 \%$ & $70 \%$ & $80 \%$ \\
$10.0 \%$ & $100 \%$ & $80 \%$ \\
$15.0 \%$ & $100 \%$ & $60 \%$ \\
$20.0 \%$ & $100 \%$ & $30 \%$ \\
\hline
\end{tabular}

Sensitivity = percentage of correct ventricular tachycardia (with atrial fibrillation or flutter) detection; Specificity = percentage of correct atrial fibrillation or flutter detection.
Table III.

Results of Method 2

\begin{tabular}{ccc}
\hline $\begin{array}{c}\text { Regularity and } \\
\text { Multiplicity Threshold }\end{array}$ & Sensitivity & Specificity \\
\hline $2.5 \%$ & $0 \%$ & $100 \%$ \\
$5.0 \%$ & $50 \%$ & $100 \%$ \\
$7.5 \%$ & $80 \%$ & $100 \%$ \\
$10.0 \%$ & $90 \%$ & $90 \%$ \\
$15.0 \%$ & $90 \%$ & $70 \%$ \\
$20.0 \%$ & $90 \%$ & $40 \%$ \\
\hline
\end{tabular}

Sensitivity = percentage of correct ventricular tachycardia (with atrial fibrillation or flutter) detection; Specificity = percentage of correct atrial fibrillation or flutter detection.

as VT. Specifically, the difference in performance between Method 1 and 2 for VT detection consisted of a single case of VT with atrial and ventricular cycle lengths close to a $2: 1$ ratio that satisfied the multiplicity criterion. The multiplicity rule improved atrial fibrillation/flutter detection by correctly identifying the atrial flutter case with 2:1 conduction but failed for atrial/fibrillation while decreasing VT detection performance in a case of VT with atrial and ventricular cycle lengths close to a $2: 1$ ratio). Therefore, the multiplicity criterion had mixed results in improving discrimination between VT and atrial fibrillation/flutter from Method 1, trading accuracy in atrial fibrillation diagnosis for VT detection accuracy. To improve the performance of Method 1 and Method 2, it is possible that the change in ventricular waveform (morphology) might need to be examined to distinguish between VT and atrial fibrillation/flutter. ${ }^{15,17,18}$

There is no statistically significant difference in performance of the two methods based upon receiver operator characteristic curve comparison. ${ }^{19}$ However, if one applied the fail-safe criterion of not missing VT as the most important performance measure, Method 1 has a better performance record for consideration for implantable devices. It must be cautioned that this is a preliminary study with only 20 cases, and more conclusive remarks must await results from larger patient population.

Although it is expected that the solution to the distinction of competing atrial and ventricular tachycardias will be a major contribution to the design of future ICDs, the presence of atrial fibrilla- 
tion with fast ventricular response remains the single most common problem in false detection in currently available devices. It is estimated that up to $60 \%{ }^{10}$ of unnecessary device therapy is due to this error. Both the Medtronics PCD ${ }^{\text {TM }}$ (Medtronic, Inc., Minneapolis, MN, USA) and the Intermedics ATD (Freeport, TX, USA) device use rate stability schemes similar to the stated "regularity" measure of this study to combat this problem. However, due to the lack of public access to software and hardware for these devices, comparison of these "regularity" measures is impossible. Therefore, the conclusion of whether the two introduced Methods would perform better than current ICD regularity schemes in the prevention of unnecessary therapy by correct identification of atrial fibrillation/flutter remains to be examined. However, the authors speculate that since the median cycle is more robust to outliers of rate than the mean or individual cycle, Method 1 should perform at least as well as the commercial "stability" measures.

The key advantage of the two methods in this study over current ICD schemes is that they incorporate atrial sensing to greatly improve the specificity of arrhythmia detection (accurate diagnosis of SVTs such as atrial fibrillation and/or flutter). This will become important for future generation ICD devices that would have separate treatment for atrial fibrillation or flutter (such as a cardioverter in the atrial channel).

This study has several limitations. The performance of the two proposed methods depended upon accurate cardiac depolarization detection, and this presumed a reliable trigger. Because the

\section{References}

1. Mower M. Cardiac Pacemakers Inc., December 1993, personnal communication.

2. Olson W. Medtronic, Inc., March 1993, personal communication.

3. Fromer M, Brachmann J, Block M. Efficacy of automated multimodal device therapy for vcntricular tachyarrhythmias as delivered by a new implantable pacing cardoverter-defibrillator. Circulation 1992; 86:363-374.

4. Haluska E, Whistler S, Calfee R. A hierarchical approach to the treatment of ventricular tachycardias. PACE 1986; 9:1320-1324.

5. Pless BD, Sweeney M. Discrimination of supraventricular tachycardia from sinus tachycardia of overlapping cycle length. PACE 1984; 7:1318-1324. intent of the present study was to test the performance of the proposed methods rather than trigger efficacy, passages where errors occurred due to missed or false triggering were manually reexamined to see if the methods would have performed if the trigger had functioned properly. Triggering for accurate cardiac event detection remains a key problem that needs to be addressed in future research. In this study, trigger errors were eliminated from analysis. Additional testing of a larger patient population may help to further refine the role of multiplicity and regularity measures for the detection of VT during atrial fibrillation or flutter.

The testing was conducted on patient recordings during electrophysiology studies in which patients were lying supine and sedated. Catheter electrodes, which are temporary, may exhibit different characteristics from chronic implanted leads. ${ }^{20}$ Whether the results would be similar in active patients needs to be examined. Bipolar lead signals only were examined for this study. Whether unipolar or other lead configurations may be superior remains to be determined.

This study represents an attempt to apply an integrated approach to two-channel analysis that examines interchannel relationships and sorts out competing (independent) rhythms versus atrial initiated ventricular conduction. The logic is simple but appears to perform well.

Two-chamber analysis of arrhythmias may provide improved discrimination of VT during atrial fibrillation or flutter. Value-added analysis using heuristics may further delineate competing rhythms by assessment of regularity of cycle length and interchamber relationships.

6. Chapman P, Troup P. The automatic implantable cardioverter-defibrillator: Evaluating suspected inappropriate shocks. J Am Coll Cardiol 1987; 7: 1075-1078.

7. Kim S, Furman S, Matos J, et al. Automatic implantable cardioverter defibrillator: Inadvertent discharges during permanent pacemaker magnet tests. PACE 1987; 10:579-582.

8. Manz M, Gerckens U, Luderitz B. Erroneous discharge from an implanted automatic defibrillator during supraventricular tachyarrhythmia induced ventricular fibrillation. Am J Cardiol 1986; 57: 343-344.

9. Steinberg J, Sugalski J. Cardiac rhythm precipitating automatic implantable cardioverter-defibrillator 
discharges in outpatients as detected from transtelephonic electrocardiographic recordings. Am J Cardiol 1991; 67:95-97.

10. Grimm W, Flores B, Marchlinski F, Electrocardiographically documented unnecessary, spontaneous shocks in 241 patients with implantable cardioverter defibrillators. PACE 1992; 15:1667-1673.

11. Newman D, Dorian P, Downar E, et al. Use of telemetry functions in the assessment of implanted antitachycardia device efficacy. Am J Cardiol 1992; 70: 616-621.

12. Sulke N, Holt P, Bostock J, et al. Inappropriate discharges by the implantable cardioverter during postoperative testing: Implications for intraoperative assessment. PACE 1990; 13:1123-1126.

13. Cohen $\mathrm{T}$, Chien $\mathrm{W}$, Lurie $\mathrm{K}$, et al. Implantable cardioverter defibrillator proarrhythmia: Case report and review of the literature. PACE 1991; 14: 1326-1329.

14. Arzbaecher R, Bump T, Jenkins J, et al. Automatic tachycardia recognition. PACE 1984; 7:541-547.

15. Chiang CJ, Jenkins JM, DiCarlo LA. An innovative two-channel rate and morphology method for complex arrhythmia diagnosis in implantable devices. 14th Annual International Conference of the IEEE Engineering in Medicine and Biology Society, 1992, pp. 496-497.

16. McDonald R, Jenkins J, Arzbaecher R, et al. A software trigger for intracardiac waveform detection with automatic threshold adjustment. IEEE Computers in Cardiology. New York, NY, IEEE Press, 1989, pp. 167-170.

17. Lin D, DiCarlo L, Jenkins J. Identification of ventricular tachycardia using intracavitary ventricular electrograms: Analysis of time and frequency domain patterns. PACE 1988; 11:1592-1605.

18. DiCarlo L, Throne R, Jenkins J. A time-domain analysis of intracardiac electrograms for arrhythmia detection. PACE 1991; 14:329-336.

19. Chiang CJ, Jenkins J, DiCarlo L. Median filtering as a sudden onset criterion to separate sinus tachycardia from ventricular tachycardia. IEEE Computers in Cardiology. New York, NY, IEEE Press, 1992, pp. 379-382.

20. Jenkins J, Noh K, Guezennec A, et al. Diagnosis of atrial fibrillation using electrograms from chronic leads: Evaluation of computer algorithms. PACE $1988 ; 11: 622-631$. 
This document is a scanned copy of a printed document. No warranty is given about the accuracy of the copy. Users should refer to the original published version of the material. 